Gynäkologische Endokrinologie 2010 · 8:97-97 DOI 10.1007/s10304-009-0347-0

Online publiziert: 21. April 2010

๑) Springer-Verlag 2010

\section{T. Strowitzki $i^{1}$ M. Ludwig ${ }^{2}$}

${ }^{1}$ Abteilung für Gynäkologische Endokrinologie und

Fertilitätsstörungen, Universitäts-Frauenklinik, Heidelberg

${ }^{2}$ Endokrinologikum Hamburg, Hamburg

\title{
Mythen in der Reproduktionsmedizin
}

„Mythen“, meint man, haben in der modernen Kinderwunschdiagnostik und therapie nichts zu suchen. Dennoch sind sie allgegenwärtig. Wir haben uns in diesem Heft mit einer Gruppe von Autoren verschiedener Themen angenommen, die immer wieder diskutiert werden, für die aber eine Evidenz in der Literatur weitestgehend fehlt. Bei anderen Themen gibt es zwar ausreichend Evidenz, allerdings wird in der täglichen Praxis häufig davon abweichend verfahren. So wird gerade im Rahmen des Embryotransfers und in der Lutealphase auf verschiedenste Weisen therapiert oder unterstützt ohne dass ausreichend Beweise vorlägen, dass dies tatsächlich hilfreich wäre. Andere Anwendungen wiederum sind in ihrer Wirksamkeit widerlegt - werden aber dennoch angeboten und sind integraler Bestandteil der Protokolle in vielen Kinderwunschzentren.

Was ist eine Gelbkörperschwäche was ist ein LUF-Syndrom? Beides sind häufige Diagnosen, dennoch sind die Diagnosekriterien vielfältig und unterschiedlich, ebenso die Therapieansätze. Mit diesen Fragen beschäftigen sich 2 der Beiträge in diesem Heft.

Häufig bekommen Kinderwunschpatientinnen den Rat, Stress abzubauen und „den Kopf freizukriegen“, um leichter schwanger zu werden. Was ist dran an diesem und anderen Mythen rund um den Kinderwunsch?

Abschließend konnten wir die renommierte Bonner Arbeitsgruppe gewinnen, zum Thema Aneuploidiescreening zu schreiben. Technisch überzeugend wie sie ist, konnten doch bisher Studien nicht belegen, dass damit tatsächlich ei- ne Verbesserung der Schwangerschaftsraten und Geburtenraten erzielbar wäre. Ist es ein Problem der Studien? Wurde die Polkörperdiagnostik bisher nicht ausreichend untersucht?

Viel zu häufig glauben wir in der täglichen Praxis unserem Bauch lieber als den Studien. Dieses Heft möchte helfen, für einige Fragestellungen Wegweiser $\mathrm{zu}$ etablieren.

Wir wünschen viel Freude beim Lesen.

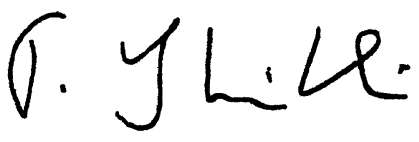

Prof. Dr. Thomas Strowitzki

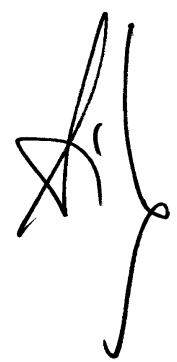

Prof. Dr. Michael Ludwig

\section{Korrespondenzadresse \\ Prof. Dr. T. Strowitzki}

Abteilung für Gynäkologische Endokrinologie und Fertilitätsstörungen, Universitäts-Frauenklinik Voßstr. 9, 69115 Heidelberg

Thomas.Strowitzki@med.uni-heidelberg.de 